\title{
Metastability and Multistability: Gelation and Liquid Crystal Formation in Suspensions of Colloidal Rods ${ }^{\dagger}$
}

\author{
M. P. B. van Bruggen \\ Philips Research, Prof. Holstlaan 4, 5656 AA Eindhoven, The Netherlands \\ H. N. W. Lekkerkerker* \\ Van't H off laboratory for Physical and Colloid Chemistry, Debye Institute, Utrecht University, \\ Padualaan 8, $3584 \mathrm{CH}$ Utrecht, The Netherlands \\ Received February 4, 2002. In Final Form: May 7, 2002

\begin{abstract}
The competition between gelation and isotropic-nematic $(\mathrm{I}-\mathrm{N})$ phase separation in dispersions of moderately attractive sterically stabilized boehmite $(\mathrm{AlOOH})$ rods is studied. Two different scenarios are observed. I n onesystem I - N phase separation proceeds by sl ow sedimentation (syneresis) of a gel resulting in a weakly birefringent, highly viscous nematic phase. In another system, closely related to the first, eventually two distinctly different nematic phases appear. One of them is again weakly birefringent and highly viscous such as in the first system. The other one is strongly bir efringent and has a lower viscosity. We present a possible explanation for the observed three-phase isotropic-nematic-nematic coexistence in terms of a combination of short-range attraction and polydispersity.
\end{abstract}

\section{Introduction}

Transitions from the liquid (isotropic) state to a liquid crystalline (nematic) state in suspensions of rodlike and platelike particles were al ready observed in the first part of the 20th century. Zocher ${ }^{1}$ was the first to observe such atransition in suspensions of vanadium pentoxideribbons. Later Bawden et al. ${ }^{2}$ and Langmuir ${ }^{3}$ observed the I-N transition in suspensions of rodlike tobacco mosaic virus (TMV) particles and platelike montmorillonite clay particles, respectively. Onsager, ${ }^{4,5}$ inspired by these observations, demonstrated that thestability of thenematic phase can beexpl ained on a purel y entropi cbasis by considering the competition between orientational entropy (favoring the isotropic state) and excluded volume entropy (which favors the nematic state). As the latter becomes more important at higher concentrations, a first-order phase transition to a nematic phase may appear if the concentration of rods or plates is sufficiently high. Since the discovery of a nematic phase in suspensions of $\mathrm{V}_{2} \mathrm{O}_{5}$, TMV, and montmorillonite, several other suspensions of rods such as suspensions of cellulose microcrystals, ${ }^{6}$ boehmite (AlOOH) rods, ${ }^{7}$ and poly(tertafluoroethylene) rods $^{8}$ have been found to exhibit the I-N transition. The $\mathrm{I}-\mathrm{N}$ transition in these aqueous suspensions is strongly salt dependent. Increase of the salt concentration leads to an increase of the concentration of rods required for the appearance of the nematic phase. This phenomenon was al ready accounted for by Onsager ${ }^{5}$ on the basis of el ectric double layer repulsion, leading to a salt-dependent ef-

† This article is part of the special issue of Langmuir devoted to the emerging field of self-assembled fibrillar networks.

(1) Zocher, H. Z. Anorg. Allg. Chem. 1925, 147, 91.

(2) Bawden, F. C.; Pirie, N. W.; Bernal, J . D.; Fankuchen, I. Nature 1936, $138,1051$.

(3) Langmuir, I. J . Chem. Phys. 1938, 6, 873.

(4) Onsager, L. Phys. Rev. 1942, 62, 558.

(5) Onsager, L. Ann. N. Y. Acad. Sci. 1949, 51, 627.

(6) Marchessault, R. H.; Morehead, F. F.; Walter, N. M. Nature 1959 184, 632.

(7) Bugosh, J . J . Phys. Chem. 1961, 65, 1789. 55 .

(8) Folda, T.; Hoffmann, H.; Chanzy, H.; Smith, P. Nature1988, 333, fectivediameter of the particles. Abovesalt concentrations of about $0.05 \mathrm{M}$, generally gelation rather than macroscopic I-N phase separation takes place. ${ }^{9-14}$ Long ago Bernal and Fankuchen ${ }^{11}$ introduced the notion of a network of clusters of parallel rods to account for the ubiquity of gelation in suspensions of rods. It has been argued $^{15}$ that van der Waals attraction can induce such a parallel aggregation of the rods. Gelation abovea critical salt concentration can then naturally be explained by the screening of theel ectrical doublelayer repulsion. ${ }^{16}$ Liquid crystallinity versus gelation however is a subtle effect and depends not only on salt concentration but also on salt composition and rod length. ${ }^{17}$

About 10 years ago Buining et al. ${ }^{18}$ synthesized for the first time sterically stabilized rodlike particles. These particles, which have a boehmite core and a steric stabilization layer of polyisobutylene, can have approximately hard core interactions ${ }^{19,20}$ but may also display moderate attractive interactions. ${ }^{21}$ By tuning the interactions between these two limits, the richness in phase behavior resulting from the competition between liquid crystallinity and gelation can be explored.

(9) J ochims, J. Kolloid Z. 1927, 41, 215

(10) Zocher, H.; J acobson, K. Kolloid-Beih. 1929, 28, 1

(11) Bernal J. D.; Fankuchen, I. J . Gen. Physiol. 1941, 25, 111.

(12) Edelson, M. R.; Hermans, J .J . Polym. Sci., Part C: Polym. Symp. $1963,2,145$

(13) Buining, P. A.; Philipse, A. P.; Lekkerkerker, H. N. W. Langmuir 1994, 10, 2106.

(14) Wierenga, A. M.; Philipse, A. P.; Lekkerkerker, H. N. W. Langmuir 1998, 14, 55.

(15) Van der Schoot, P. J . Phys. Chem. 1992, 96, 6083.

(16) Verwey, E. J . W.; Overbeek, J . Th. G. Theory of the stability of Iyophobic colloids; Elsevier: Amsterdam, 1948

(17) Borgström, J.; Egermayer, M.; Sparrman, T.; Quist, P. O.; Piculell, L. Langmuir 1998, 14, 4935.

(18) Buining, P. A.; Veldhuizen, Y. S. J .; Pathmamanoharan, C. Lekkerkerker, H. N. W. Colloids Surf. 1992, 64, 47.

(19) Buining, P. A.; Lekkerkerker, H. N. W. J . Phys. Chem. 1993, 97, 11510.

(20) Van Bruggen, M. P. B.; Dhont, J . K. G.; Lekkerkerker, H. N. W. Macromolecules 1999, 32, 2256.

(21) Van Bruggen, M. P. B.; Van der Kooij, F. M.; Lekkerkerker, H. N. W. J . Phys.: Condens. Matter 1996, 8, 9451. 
In this study the coexistence of two nematic phases together with an isotropic phase is reported. Although the origin of the attractions has not yet been unraveled in these suspensions, its effect is unquestionable since one of the coexisting nematics consists of aggregated birefringent clusters. We indicate how a combination of attractive interactions together with polydispersity of the colloidal particles may lead to the observed phenomena.

Theoutline of this articleis as follows. In thenext section experimental details are given about the preparation of the colloidal systems and the experimental equipment used. In section 3 the observations are presented which are discussed in section 4. The conclusions aresummarized in section 5 .

\section{Experimental Section}

2.1. Polyisobutylene Grafted Boehmite Rods. Aqueous di spersions of charge-stabilized boehmite rods weretransferred to 1-propanol and grafted with modified polyisobutylene (PIB). The synthesis of boehmite rods and the grafting procedure are described in ref 18. The PIB (provided by Shell) consists of a polyalkylamine anchor group which is chemically connected to two polyisobutylene tails with a molecular weight of $1000 \mathrm{~g} / \mathrm{mol}$ each. ThePIB grafted rods were dispersed in cyclohexane(M erck, p.a). Thesystem BPIB (b) was obtained by distilling BPIB (a) after 1 year of standing to pure cyclohexane. Dispersions were concentrated by low-speed $(2500 \mathrm{rpm})$ centrifugation and re dispersion of the sediment. The dimensions of the rods were determined with transmission el ectron microscopy. Theaverage length of the rods is $190 \mathrm{~nm}$, and the average diameter is $17 \mathrm{~nm}$ (including the PIB chains). The polydispersity of the sterically stabilized rods was determined from a large number of transmission el ectron microscopy photographs. The polydispersity was found to be $25 \%$ in both length and diameter. The volumefraction of rods is corrected for the fact that the PIB chains encl osesolvent. When the rods are much longer than the length, $h$, of the PIB chains, the corrected volume fraction $\phi$ is given by

$$
\phi=\frac{\mathrm{C}}{\rho_{\mathrm{b}}(1+\mathrm{x})}\left(\frac{\mathrm{D}+2 \mathrm{~h}}{\mathrm{D}}\right)^{2}
$$

where $\mathrm{c}$ is the mass concentration of the PIB grafted boehmite rods in the dispersion, $D$ is the diameter of the rods, $\rho_{b}$ is the density of the bare boehmite rods $(3.0 \mathrm{~g} / \mathrm{mL})$, and $x$ is the mass ratio of PIB to boehmite (typically 0.25 ).

2.2. Polarization Light Microscopy. The phase separation processes in both samples were studied under a polarization light microscope (Zeiss, axioplan). Test tubes containing phaseseparating samples were vortexed and transferred into glass cuvettes (Vitrodynamics) with an optical path of $100 \mu \mathrm{m}$.

2.3. Fluorescence Confocal Scanning Laser Microscopy. Boehmite rods grafted with modified PIB have fluorescent properties which probably arisefrom the anchor group although its origin has not been clarified yet. Because of this feature, the morphology of the birefringent phases could also investigated with fluorescence confocal scanning laser microscopy (CSLM). Details about the experimental setup can be found el sewhere.22 With this technique structures can be analyzed in a focal plane with a resolution in depth of $500 \mathrm{~nm}$. This is in contrast to polarization microscopy where in-focus and out-of-focus information is superimposed. The detected local fluorescent intensity is a measurefor thelocal particledensity, instead of the local order as with polarization microscopy.

\section{Results}

3.1. BPIB(a). System BPIB(a) was used in an earlier study. ${ }^{21}$ This system starts phase separating, albeit extremely slowly (months), into two phases when the concentration was higher than $5.2 \%$. The bottom phase is birefringent whereas the upper phase is isotropic. At $12.2 \%$ the nematic phase spans the entire tube. Macro-

(22) Van Blaaderen, A.; Wilzius, P. Science 1995, 270, 1177.
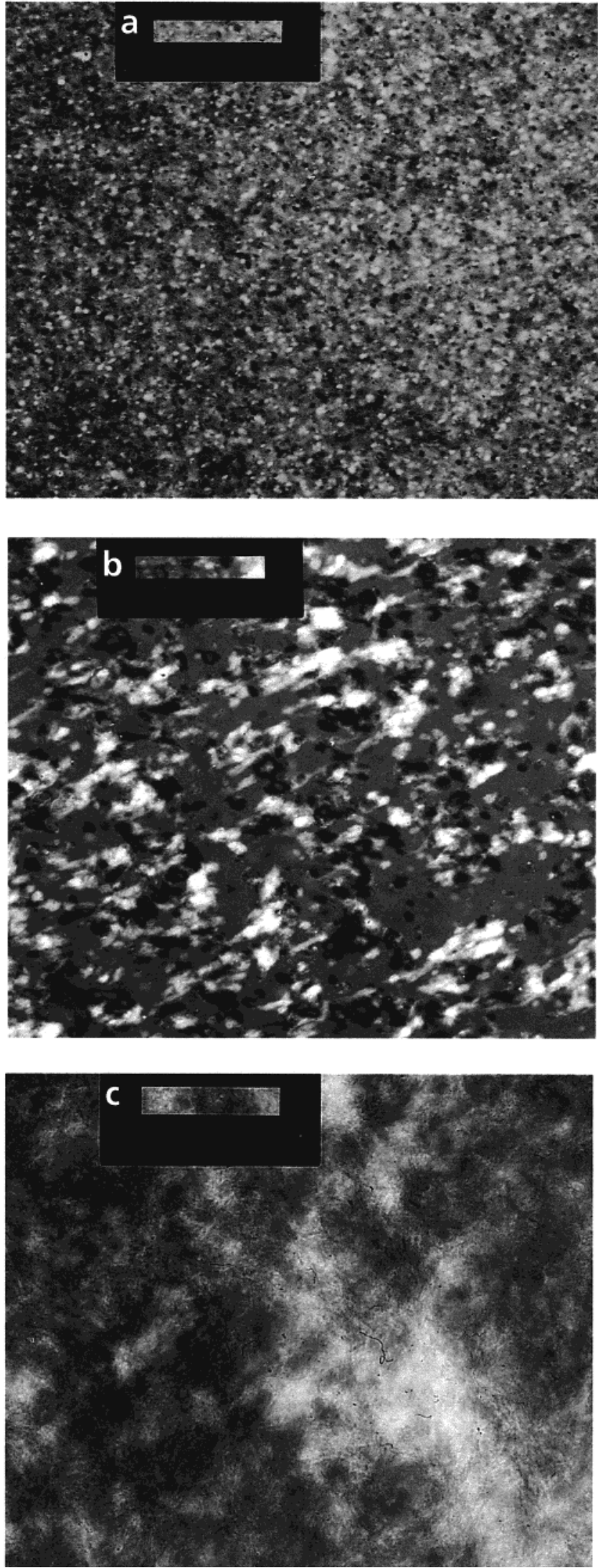

Figure 1. Polarization micrographs of a BPIB (b) sample with an overall concentration of $8.3 \%$ (a) 40 min after homogenization, (b) $20 \mathrm{~h}$ after homogenization, and (c) the bottom phase after macroscopic phase separation. The bars represent 200 $\mu \mathrm{m}$.

scopic phase separation of dispersions with a volume fraction smaller than $12.2 \%$ is attained after 1 to 2 months, 


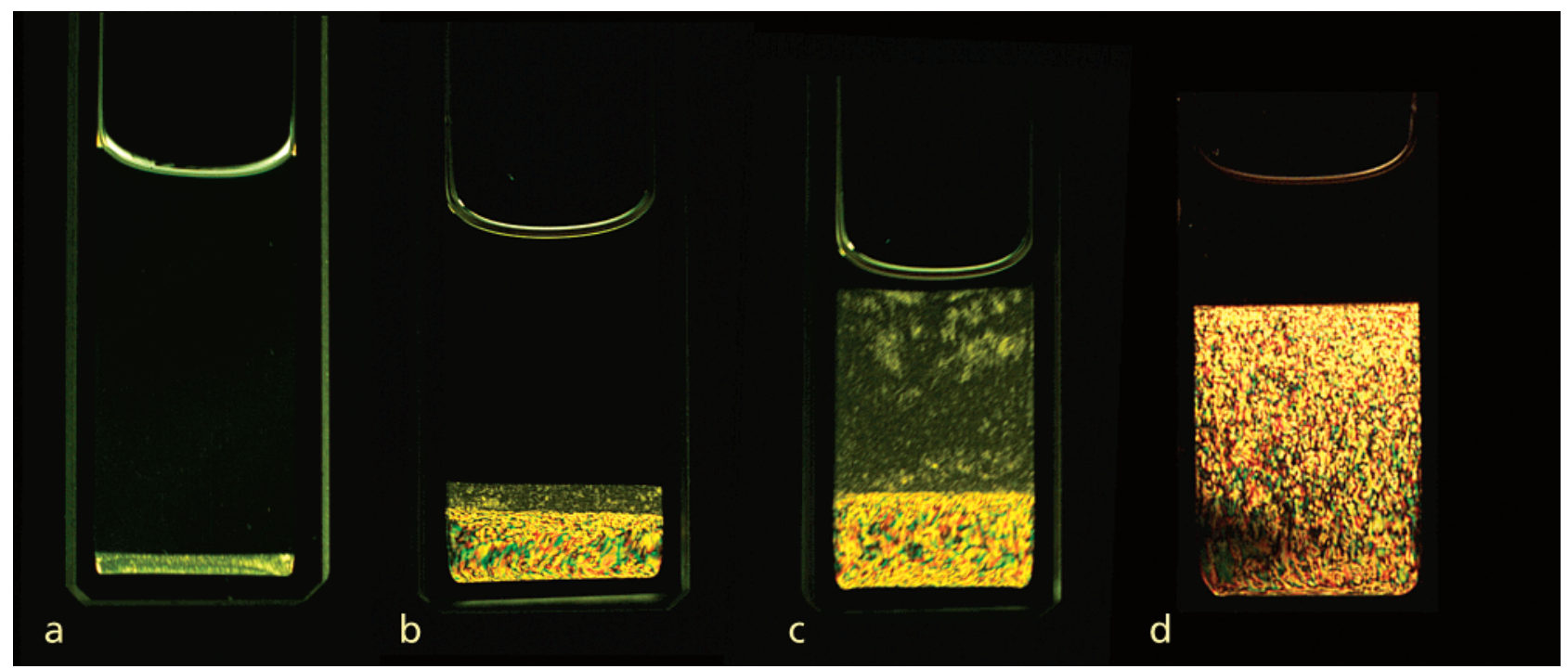

Figure 2. Cuvettes containing macroscopically phase separated BPIB(b) with an overall concentration of (a) $8.3 \%$, (b) $11.7 \%$, and (c) $15.4 \%$ between crossed polarizers and (d) macroscopically phase separated hard rod dispersion with a volume fraction of $22 \%$ between crossed polarizers.

but for the highest concentrations this lasts even longer. Phase separation is also accompanied by a small-angle light scattering ring, whose radius becomes smaller with time. After $24 \mathrm{~h}$ the ring has filled up and has become a light-scattering cone in the forward direction. This lightscattering cone remains spherical during the whole separation process. In samples with a rod concentration somewhere in the middle of the two-phase region, polarization microscopy revealed that phase separation proceeded by roughening of initiall y smooth areas, resembling spinodal decomposition. H owever this process stops when the typical domain size is only a few micrometers large, leaving a frozen in spinodal-likestructure which sediments under gravity from the meniscus. The nematic phase is weakly birefringent, very turbid, and does not flow upon tilting the tube.

3.2. BPIB(b). A rather different phase behavior is displayed by the BPIB (b) dispersion which was obtained by purification of BPIB (a) as described in section 2 . Above a volume fraction $\phi_{1}=7 \%$, the system becomes turbid in timeand starts phase separating. Thevalue of $\phi_{1}$ is higher than that found for the BPIB(a) system. The duration of macroscopic phase separation is typically a few weeks, i.e., significantly faster than that for BPIB(a). At a volume fraction of $8.3 \%$ the phase separation process of $B P I B(b)$ was followed under the polarization microscope and revealed that small birefringent droplets became visible to the eye only after $1 \mathrm{~h}$ (F igure la). After $20 \mathrm{~h}$ Figure $1 \mathrm{~b}$ shows that the droplets have assembled into large irregularly shaped domains of tens of micrometers. Figure 1c represents a polarization micrograph of the finally obtained bottom phase. The birefringence in this phase is vague, and typical nematic textures are absent. A new and surprising feature in the phase behavior shows up for volume fractions above $8.3 \%$. In a sample with a rod concentration of $11.7 \%$, no separate droplets are visible anymore but initially smooth birefringent areas start roughening in time. However after a few days, in this system droplets have been devel oped as well. Two bire fringent bottom phases are eventually formed in this sample. The bottom nematic phase is a transparent, birefringent phase exhibiting higher order birefringence colors such as in a phase-separated sample containing hard rods. ${ }^{20}$ The middle phase is a more turbid and less birefringent phase, resembling the phase formed at $8.3 \%$.

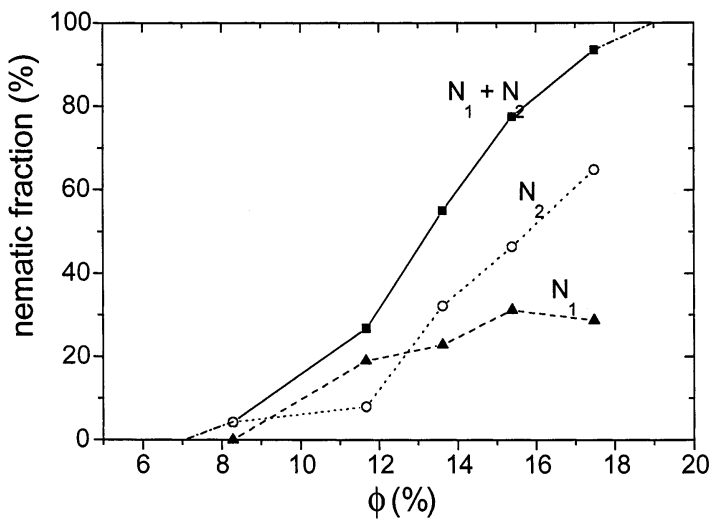

Figure 3. Phase diagram of the BPIB(b) system.

The upper phase is isotropic, exhibiting only streaming birefringence. In Figure 2 photographs of samples with increasing concentrations placed between crossed polarizers are presented. For comparison a phase-separated samplecontaining hard rods ${ }^{20}$ is presented as well. As the overall concentration is further increased the volumes of the bottom phases increase, whereas the volume of the isotropic phase decreases. The dependence of the phase volumes on the overall rod volume fraction is presented in Figure 3. Theisotropic phasecompletel y vanishes above a volume fraction of $19 \%$, and only two birefringent phases are left.

In Figure 4 a $100 \times 100 \mu \mathrm{m}$ CSLM cross section of the middle nematic phase is given of the sample with a rod concentration of $11.7 \%$. The alternating white and dark areas look remarkably similar to the polarization micrograph of this phase shown in Figure 2. The CSLM micrograph indicates that the density of rods in themiddle nematic phase is inhomogeneous. This conclusion is also drawn for clay gels from direct visualization by synchrotron-based X-ray fluorescencemicroscopy. ${ }^{23}$ I $n$ thebottom phase, as well as in thetop phasethefluorescent intensity does not vary; the density is constant in these phases. The concentration of rods in the dark areas in $\mathrm{F}$ igure 4 roughly corresponds to that of the coexisting isotropic phase,

(23) Bihannic, I. Michot, L.J .; Lartiges, B. S.; Vantelon, D. V.; Labille J . Thomas, F. Susini, J .; Salome, M.; Fayard, B. Langmuir 2001, 17, 4144. 


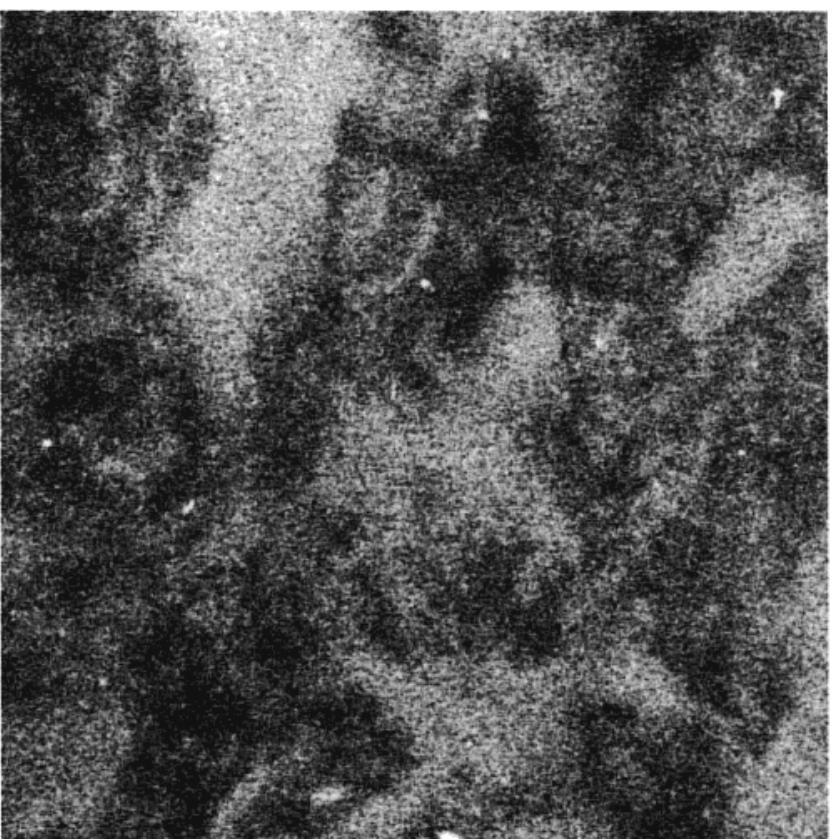

Figure 4. $100 \times 100 \mu$ m confocal fluorescencelaser micrograph of the middle nematic phase shown in Figure $2 \mathrm{~b}$. The areas which arelight correspond to high fluorescenceand thus a high density of rods. The few bright spots are dense aggregates of rods of a few micrometers in size.

whereas the light areas correspond to densities close to that of the bottom phase. This implies that when extrapolating the (N1 + N2) curve in Figure 3, the isotropic phase will be completely trapped inside the voids of the nematic network of the middle phase at a volume fraction of about $19 \%$.

\section{Discussion}

The most remarkable phenomenon is the occurrence of a three-phase equilibrium with two nematic phases, which is encountered after purification of the solvent in BPIB(a). A three-phase coexistence region in a one-component system is in contradiction to Gibbs' phase rule. In this dispersion, coded as BPIB(b), the low-density nematic appears not to be in equilibrium, in view of its inhomogeneous morphology as is revealed by CLSM. This nematic phase seems to consist of aggregates that have been clustered together. At higher concentrations a second higher density nematic phase is being formed which coexists with both a nonequilibrium nematic phase and an isotropic phase. On the basis of its morphology this nematic phase is not aggregated or gelled. In the BPIB(a) dispersion apparently one single nematic phase is being formed, but the appearance of this nematic phase resembles the nonequilibrium nematic in BPIB(b). Since short-range attractions as well as pol ydi spersity enhance nematic-nematic coexistence, ${ }^{24,27}$ wesuggest that a threephase nematic-nematic-isotropic coexistence region in the BPIB (b) system is engendered by a combination of both. Contrary to a monodisperse system, polydispersity can in principlegiverisetomultiphasecoexistenceregions such as the three-phase region observed in this study. Although the origin of the attractive interactions in this study is not clear, small solvent impurities such as water

(24) Flory, P. J . Proc. R. Soc. London 1956, A234, 73.

(25) Kohkhlov, A. R.; Semenov, A. N. J . Stat. Phys. 1985, 38, 161.

(26) Vroege, G. J .; Lekkerkerker, H. N. W. J . Phys. Chem. 1993, 97 11510 405

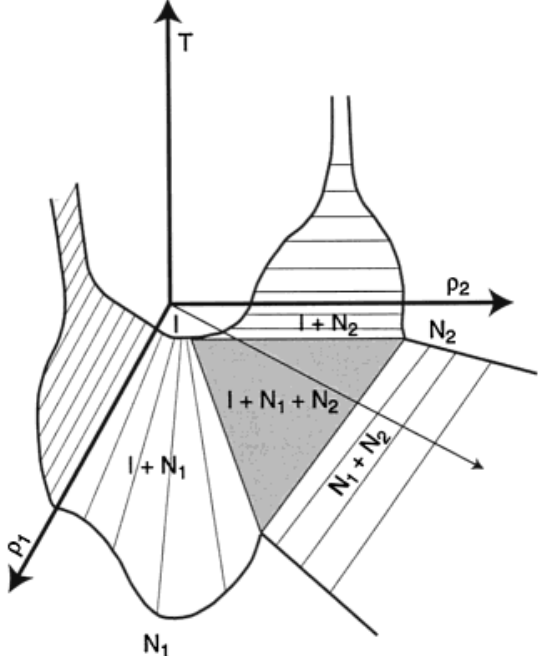

Figure 5. Proposed phase diagram of a bidisperse system of attractive rods as a function of the compostion $\rho_{1}$ and $\rho_{2}$ and the temperature $T$.

might affect the colloidal interactions. Additions of traces of water to the suspensions did however not lead to significant changes in the behavior of the suspensions. A sketch of a possible phase diagram of a bidisperse dispersion of attractive rods with length $L_{1}$ and number density $\rho_{1}$ and rods with length $L_{2}$ and number density $\rho_{2}$ is given in Figure 5 . The slope of the thin arrow in this figurecorresponds to the $\rho_{1} / \rho_{2}$ ratio present in the system. With increase of the volume fraction of an initially homogeneous and stable isotropic phase, first the nematic phase indicated by $\mathrm{N}_{2}$ will be formed, which coexists with theisotropic phase, indicated by l. After further increasing the concentration, the gray shaded area in Figure 5 is entered and a second nemati c phase, $\mathrm{N}_{1}$, is being formed. Finally the isotropic phase disappears and two coexisting nematics, $\mathrm{N}_{1}$ and $\mathrm{N}_{2}$, remain. As the two nematic phases consist of two different particle populations, phase separation would be in principle an adequate way to purify polydispersedispersions. All experiments were performed at constant temperaturetherefore specific changes of the dispersion medium determine the "temperature" of the systems under consideration. So at very high temperature (i.e., very small attractions) the particles behave as hard rods and polydispersity (here modeled as bidispersity) alone is insufficient to induce three-phase coexistence. This corresponds to the behavior as observed in hard rod dispersions as has been published elsewhere. ${ }^{20}$ For intermediate low temperatures (i.e., weak attractions) the scenario as described above can occur and BPIB(b) seems to confirm this, al beit that one of the two nematic phases has been kinetically arrested. Theeffect of a kineticar rest will be enhanced at still lower temperatures (e.g., strong attractions), and the three-phase region can become completely hidden, resulting in the coexistence of an isotropic phase and an apparent single birefringent, "nematic" phase, as observed in the BPIB(a) dispersion.

The kinetically arrested nematic phase in the BPIB(b) dispersion proceeds along the same way as has been observed in a dispersion of hard rods to which nonadsorbing polymer had been added. ${ }^{28}$ In this study it was found that in dispersions with a composition just within the two-phase region, the formed nematic phase aggregated abovea certain polymer concentration. This was

(28) Van Bruggen, M. P. B.; Lekkerkerker, H. N. W. Macromolecules $\mathbf{2 0 0 0}, 33,5532$ 
explained by the fact that the concentration of the coexisting nematic phase exceeded the glass line. The visual observations closely resemble the nucleation and subsequent aggregation of the tactoidal droplets in the $\mathrm{BPIB}(\mathrm{b})$ dispersion as shown in Figure 1. Following the same line of reasoning, the $\mathrm{N}_{2}$ nematic in the BPIB(b) system has a concentration that exceeds the glass line, whereas for the $\mathrm{N}_{1}$ nematic this is not yet the case.

\section{Summary and Conclusion}

The occurrence of a three-phase nematic-nematicisotropic equilibrium in a dispersion of sterically stabilized rods is explained on the basis of a bi disperse phasediagram of attractive rods. At intermediate attractions it is proposed that a three-phase region arises which disappears as the attractions become smaller (i.e., higher temperatures or better solvent quality). When the attractions are stronger (i.e., lower temperatures or worse solvent quality), the three-phase region remains but the concentration of the coexisting nematic phases approach the glass line. At a certain point one of the coexisting concentrations exceeds the glass line leading to aggregate-nematic-isotropi c coexistence. This is observed in the dispersion coded as BPIB(b). If the attraction becomes even stronger, both coexisting concentrations will exceed the glass line and the two aggregated phases collapse onto one apparent bi refringent phase. This type of behavior is displayed by the dispersion coded as BPIB(a).

Acknowledgment. Professor A. van Blaaderen kindly provided us with theCSLM data. This work was supported by the Council for Chemical Sciences from The Netherlands Organization for Scientific Research (NWO/CW).

LA020161B 